\title{
Use of novel immunohistochemical markers expressed in colonic adenocarcinoma to distinguish primary ovarian tumors from metastatic colorectal carcinoma
}

\author{
Sanjay Logani ${ }^{1}$, Esther Oliva ${ }^{2}$, Paula M Arnell ${ }^{2}$, Mahul B Amin ${ }^{1}$ and Robert H Young ${ }^{2}$ \\ ${ }^{1}$ Emory University School of Medicine, Atlanta, GA, USA and ${ }^{2}$ The James Homer Wright Pathology \\ Laboratories, Massachusetts General Hospital, Harvard Medical School, Boston, MA, USA
}

\begin{abstract}
Distinguishing primary ovarian carcinoma, particularly endometrioid and mucinous subtypes, from metastatic colorectal carcinoma to the ovary is often difficult on histologic examination alone. Recently, three immunohistochemical markers CDX2, a homeobox gene encoding an intestine-specific transcription factor; $\alpha$-methylacyl-CoA racemase (AMACR/P504S), a mitochondrial and peroxisomal enzyme with fairly restricted expression in selective tumors and $\beta$-catenin, an adenomatous polyposis coli (APC) mutation product resulting in activation of the Wnt pathway, have been reported to have specific and sensitive expression in colorectal carcinomas. We evaluated a panel consisting of antibodies to CDX2, $\beta$-catenin and P504S in 23 primary ovarian adenocarcinomas (13 mucinous and 10 endometrioid) and compared the findings to 22 metastatic colorectal adenocarcinomas (seven mucinous and 15 nonmucinous tumors with endometrioid-like morphology hereafter referred to as pseudo-endometrioid) to the ovary stained with the same panel. Twenty (91\%) metastatic tumors expressed at least two markers and seven $(32 \%)$ expressed all three. In contrast, only three (13\%) primary ovarian tumors expressed at least two markers and none expressed all three. Strong $(2+, 3+)$ and diffuse ( $>40 \%$ ) expression for CDX2 was noted in $21(95 \%)$ metastatic tumors and five $(22 \%)$ primary ovarian tumors (three mucinous, two endometrioid). P504S was similarly expressed in seven (32\%) metastatic and none of the primary ovarian carcinomas. Nuclear expression of $\beta$-catenin was noted in $13(59 \%)$ metastatic tumors and in eight cases (36\%), it was diffuse and strong. In contrast, four (19\%) primary tumors showed nuclear expression of this protein with only one (5\%) case expressing it in a diffuse pattern. Immunohistochemical expression of gene products and enzymes of colorectal carcinogenesis in some primary ovarian carcinomas suggest that the morphologic similarities between colorectal and mucinous/endometrioid carcinoma of the ovary extends to the genetic level, although differences in the level of expression exist between these tumors. Diffuse expression of all three markers (CDX2, $\beta$-catenin and P504S) in a tumor in the ovary was found to be virtually diagnostic of metastasis from a colorectal primary in this study.
\end{abstract}

Modern Pathology (2005) 18, 19-25, advance online publication, 24 September 2004; doi:10.1038/modpathol.3800260

Keywords: ovarian cancer; mucinous; adenocarcinoma; endometrioid adenocarcinoma; metastatic; colorectal carcinoma; CDX2; $\beta$-catenin; P504S

It has been estimated that metastatic tumors to the ovary account for approximately $7 \%$ of all ovarian tumors ${ }^{1}$ and the majority of these represent spread from primary lesions in the gastrointestinal tract. ${ }^{2}$ Distinguishing primary ovarian adenocarcinoma, particularly endometrioid and mucinous subtypes,

Correspondence: Dr S Logani, MD, Department of Pathology, Emory University Hospital Suite H187, 1364 Clifton Road, NE, Atlanta, GA 30322, USA.

E-mail: slogani@emory.edu

Received 16 June 2004; revised and accepted 16 July 2004; published online 24 September 2004 from metastatic tumors from the colon can be challenging. ${ }^{3}$ In the past decade, numerous markers with differing specificity and sensitivity have been evaluated in the above differential diagnosis. ${ }^{4-8}$ The use of differential cytokeratin expression using cytokeratin 7 (CK7) and cytokeratin 20 (CK20) in this group of tumors is of great value, although limited by the frequent expression of cytokeratin 20 in primary mucinous tumors of the ovary (reported to be up to $60 \%$ in some studies) and the coexpression of CK7 by a small subset of primary colonic adenocarcinomas. ${ }^{6,9,10}$ More recently, immunohistochemical detection of cellular proteins such as CDX2 and $\beta$-catenin in colorectal carcinoma, 
overexpressed as a consequence of genetic alteration in their respective genes, has fostered an interest in the use of antibodies to these proteins as specific markers of colorectal differentiation in a carcinoma. Recent immunohistochemical studies have shown CDX2 to be an exquisitely sensitive and highly specific marker of intestinal adenocarcinomas. ${ }^{11-13}$ Similarly, nuclear overexpression of $\beta$-catenin has been observed in a high percentage of intestinal adenocarcinomas. ${ }^{14} \quad \alpha$-Methylacyl-CoA racemase (AMACR) or P504S, an enzyme involved in bile acid biosynthesis, has been shown to be a highly sensitive marker for colorectal carcinomas, although its expression has been shown to be directly related to the grade of the tumor. ${ }^{15,16}$ The use of these antibodies in clinical practice to identify a metastatic tumor as colorectal in origin when the primary site may not be clinically apparent is of great potential and provides a useful addition to CK7 and CK20. In this study, we evaluated the utility of a panel of immunohistochemical stains, CDX2, $\beta$ catenin and P504S, in distinguishing primary mucinous and endometrioid adenocarcinoma from metastatic colorectal adenocarcinoma to the ovary.

\section{Materials and methods}

Twenty three primary ovarian tumors (13 mucinous, 10 endometrioid) and 22 metastatic colonic tumors to the ovary (7 mucinous and 15 pseudoendometrioid) were identified from the surgical pathology files of Emory University Hospital and Massachusetts General Hospital. The classification of the tumors into primary vs metastasis was reviewed using established histologic criteria ${ }^{3,17}$ and in cases of putative primary tumors where morphologic findings were not diagnostic, clinical records were reviewed to confirm absence of a gastrointestinal primary on follow-up.

Representative hematoxylin and eosin stained slides from the tumors were reviewed and one representative block selected for immunohistochemical staining. Immunohistochemical studies for CDX2 (CDX2-88, 1:200, BioGenex, San Ramon, CA, USA), P504S (Z2001, 1:40, Zeta Corp., Sierra Madre, CA, USA) and $\beta$-catenin (E-5, 1:80, Santa Cruz, Santa Cruz, CA, USA) were performed. Data from $\beta$ catenin staining performed on some of the primary ovarian tumors and metastatic colonic tumors was presented in abstract form previously and included in the current study. ${ }^{18}$ Briefly, $5 \mu \mathrm{m}$ sections were deparaffinized and rehydrated. Antigen retrieval was performed by pressure cooking the slides in citrate buffer ( $\mathrm{pH}$ 6) for $5 \mathrm{~min}$ at $120^{\circ} \mathrm{C}$. All the tissue was then exposed to $3 \%$ hydrogen peroxide for 5 min (to block endogenous biotin reactivity), primary antibody for $25 \mathrm{~min}$, biotinylated secondary antibody for $25 \mathrm{~min}$, streptavidin enzyme complex for $25 \mathrm{~min}$, diaminobenzidine as a chromogen for $5 \mathrm{~min}$ and hematoxylin counterstain for $5 \mathrm{~min}$. All immunohistochemical staining was performed by an automatic stainer (DAKO, Carpinteria, CA, USA). Stained slides were then coverslipped using the Tissue-Tek.SCA (Sakura finetek, USA, Inc., Torrance, CA, USA) automatic coverslipper. Appropriate positive and negative controls were included.

Extent of immunohistochemical expression was recorded and scored as $0,<10 \% ; 1+, 11-40 \% ; 2+$, $41-70 \%$ and $3+, 71-100 \%$. In addition, intensity of staining was also recorded and scored on a threetiered system as $1+$ : weak, $2+$ : moderate and $3+$ : strong. Nuclear expression of CDX2, cytoplasmic expression of P504S and both nuclear and membranous expression for $\beta$-catenin was recorded.

\section{Results}

The extent of positivity for CDX2, P504S and $\beta$ catenin (nuclear) is summarized in Table 1.

\section{Metastatic Colonic Adenocarcinoma $(n=22)$}

Moderate to strong $(2+, 3+)$ and diffuse $(3+)$ nuclear expression for CDX2 was noted in all but one of the tumors (Figure 1a). There was no correlation observed between the extent of CDX2 expression and tumor type (mucinous vs pseudoendometrioid) and the grade of the tumor. Seven of 22 $(32 \%)$ cases showed expression for P504S in greater than $40 \%$ of the cells $(2+$ to $3+)$ that was moderate to strong in intensity (Figure 1b). Nuclear expression for $\beta$-catenin $(2+, 3+)$ was noted in $36 \%$ of the tumors (Figure 1c), predominantly cytoplasmicmembranous pattern was noted in $55 \%$ of cases and in two cases $(11 \%)$, the staining pattern was both membranous and nuclear. Twenty tumors expressed at least two markers and seven showed expression of all three markers.

\section{Primary Ovarian Adenocarcinoma $(n=23)$}

CDX2 was typically expressed as weak/focal nuclear staining in the majority of the cases (Figure 2),

Table 1 Extent of positivity for CDX2, P504S and $\beta$-catenin expression

CDX2: 1+ CDX2: 2+, 3+ P504S: 1+ P504S: 2+, 3+ $\beta$-Catenin (nuc): 1+ $\beta$-Catenin (nuc): 2+, 3+

Primary ovarian carcinoma $n=23$

Metastatic colonic carcinoma $n=22$

$26 \%$

$4.5 \%$

$22 \%$

$95 \%$
$13 \%$

$9.5 \%$

\section{$0 \%$}

$32 \%$
$14 \%$

$23 \%$
$5 \%$

$36 \%$ 

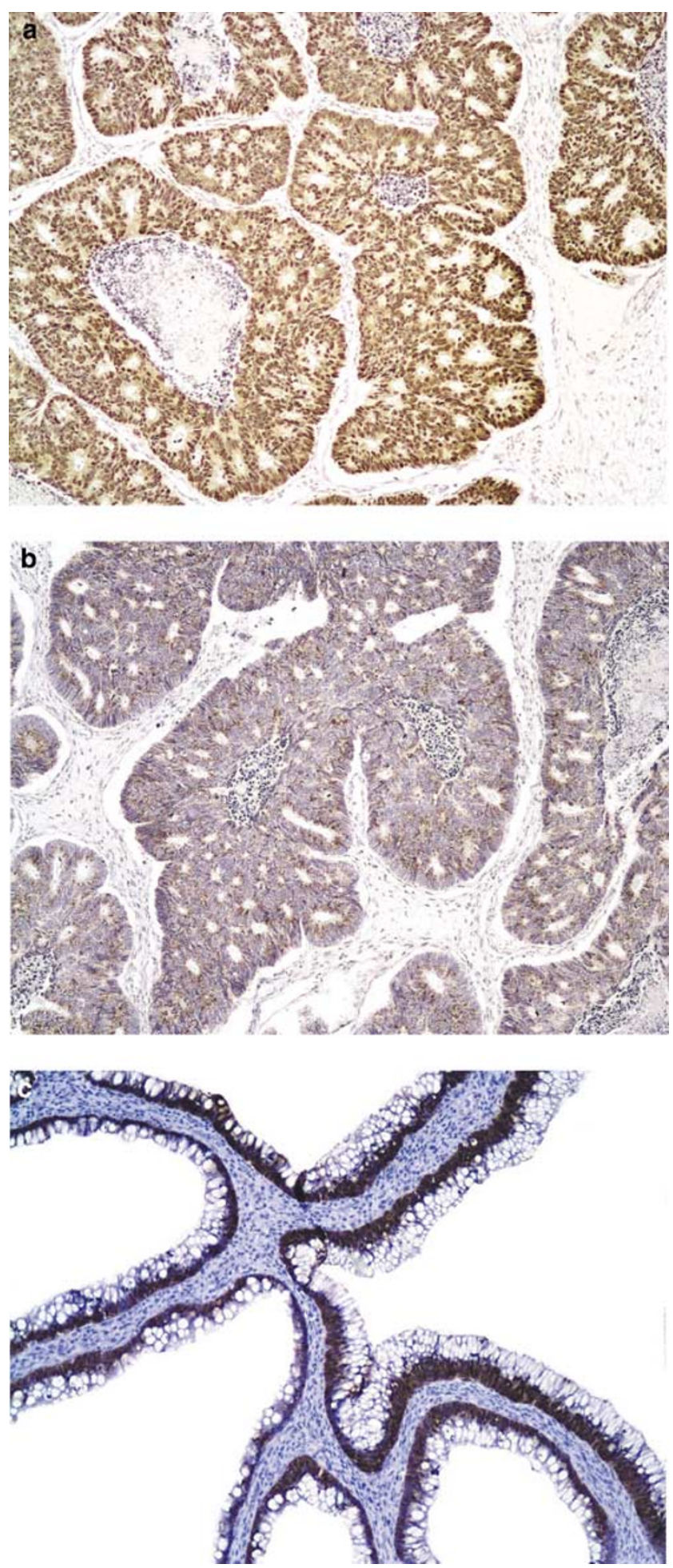

Figure 1 Typical pattern of expression for CDX2 (a), P504S (b) and $\beta$-catenin (c) in metastatic colorectal carcinoma to the ovary. Note the differentiated nature of the mucinous epithelium in (c). Diffuse and strong nuclear expression for CDX2 and $\beta$-catenin was observed in metastatic tumors.

however, five of 23 cases (22\%) showed diffuse $(2+$, $3+$ ) nuclear expression of CDX2. Three were mucinous and two endometrioid in histology. None

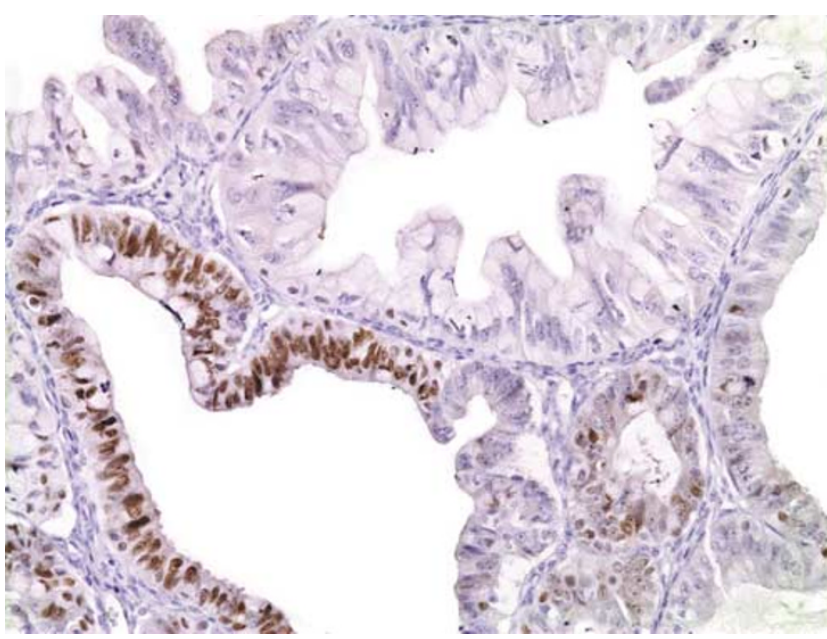

Figure 2 CDX2 expression in primary mucinous ovarian adenocarcinoma. Although, strong and diffuse staining was observed in approximately $20 \%$ of tumors in this group, the typical pattern of expression was either negative or focally positive as seen here.

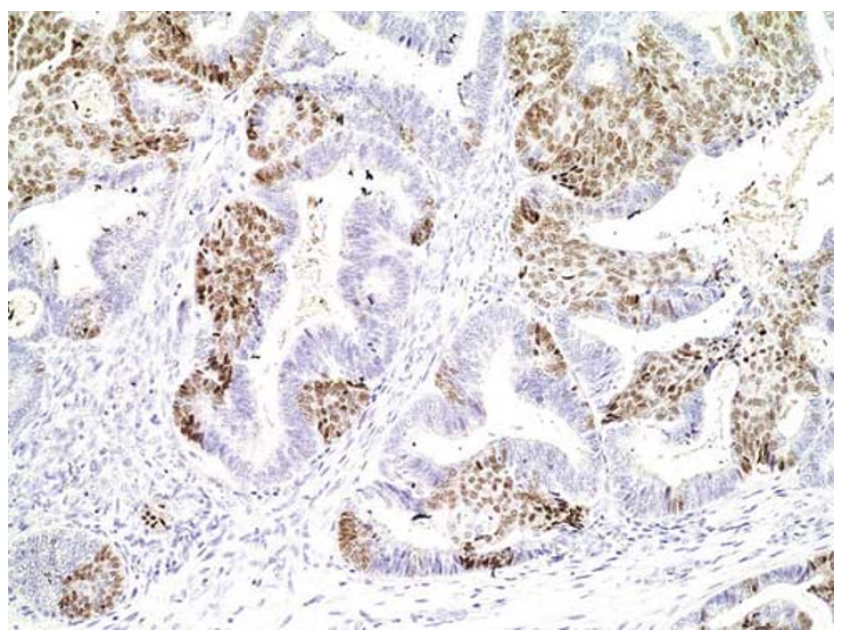

Figure 3 Strong nuclear expression for CDX2 in foci of squamous metaplasia in a primary endometrioid adenocarcinoma of the ovary.

of the tumors expressed P504S $(2+, 3+)$ and nuclear staining for $\beta$-catenin $(2+, 3+)$ was observed in $5 \%$ of the tumors. In two endometrioid tumors with extensive squamous metaplasia, strong nuclear staining with $\beta$-catenin was observed in foci of squamous metaplasia (Figure 3). Diffuse staining with at least two markers was observed in only three tumors and none of the tumors expressed all three markers.

\section{Discussion}

Colorectal adenocarcinoma is the most frequent metastatic tumor to the ovary that mimics a primary 
Mullerian adenocarcinoma, usually one of endometrioid or mucinous cell type..$^{2,17,19,20}$ This important distinction has clinical implications for accurate staging of the tumor as well as for therapeutic options. Recent studies have highlighted the salient morphologic features helpful in distinguishing a primary from a metastatic tumor, ${ }^{3,20}$ however, occasionally immunohistochemical studies may be very helpful as supportive evidence in the above differential diagnosis. Differential staining with CK7 and CK20 in conjunction with morphology and clinical information is usually adequate in ascribing a primary site in the majority of the $\operatorname{cases}^{4,6,7,9,10}$ however, a small percentage of primary ovarian adenocarcinomas, most often mucinous or endometrioid subtypes may be difficult to distinguish from a colorectal metastasis using differential cytokeratin expression. This is largely in part due to the high percentage (approximately $60 \%$ in one study) of primary mucinous ovarian tumors that may express cytokeratin $20^{4,6,9}$ and coexpression of CK7 in up to $10 \%$ of colorectal tumors. ${ }^{10}$ The recent development of immunohistochemical markers to proteins that are specifically upregulated in colorectal carcinoma has provided another diagnostic tool that may be useful in the small percentage of cases where distinguishing a primary ovarian from a colorectal metastasis may be problematic using conventional morphologic criteria alone. In this study, we focus on the utility of three novel markers expressed in colorectal carcinoma, CDX2, $\beta$-catenin and P504S, to be used as a potential panel in distinguishing between a primary carcinoma and a colorectal carcinoma metastatic to the ovary.

Human CDX2 protein is a member of the homeobox gene that encodes an intestine-specific transcription factor. ${ }^{21,22}$ This protein regulates normal intestinal development and is expressed in the nuclei of epithelial cells throughout the intestinal tract in embryonic and postnatal life. Expression of CDX2 mRNA has been shown to be highly restricted to intestinal epithelium. ${ }^{23}$ The sensitivity and specificity of antibodies to CDX2 protein as a marker of colonic adenocarcinoma has been recently evaluated in a few studies with reported sensitivity and specificity of greater than $90 \% .{ }^{11-13}$ In our study, $95 \%$ of the metastatic colorectal carcinomas to the ovary showed CDX2 expression. The staining pattern in all the cases was uniform with strong nuclear expression in almost all nuclei and was independent of the tumor subtype (mucinous vs pseudoendometrioid). CDX2 expression in a pattern similar to that observed in colonic metastasis was observed in five $(22 \%)$ of primary ovarian adenocarcinomas. These tumors represented $21 \%$ of the mucinous ovarian carcinomas and $20 \%$ of the endometrioid tumors evaluated in this study. An interesting observation within the mucinous subtype was the complete absence of CDX2 expression in primary ovarian tumors with an expansile pattern of invasion. Morphologically, an expansile pattern has been reported to be present in $88 \%$ of primary ovarian mucinous carcinomas and $18 \%$ of metastatic tumors in one recent study. ${ }^{3}$ In contrast, the three mucinous tumors with strong CDX2 expression showed an infiltrative pattern of growth characterized by small glands irregularly infiltrating the ovarian stroma including one with extravasated mucin in the stroma (pseudomyxoma ovarii). Since this morphologic pattern of invasion is more commonly observed in metastatic tumors, ${ }^{3}$ clinical correlation is essential in these difficult cases; in the absence of any clinical evidence of a colonic primary, the strong expression of CDX2 in these cases likely represents a true enteric phenotype in the primary ovarian neoplasm. There were two primary endometrioid tumors in which strong CDX2 expression was observed in a pattern indistinguishable from that observed in metastatic colorectal carcinoma. One of these cases also showed strong nuclear expression for $\beta$-catenin, but neither expressed P504S thus illustrating the importance of using an immunohistochemical panel in this differential diagnosis. Moskaluk et $a l^{13}$ found strong CDX2 expression in $20 \%$ of endometrioid and $20 \%$ of mucinous carcinomas of the ovary, results that are comparable to the current study. However, four other series comprising a total of 55 primary mucinous carcinomas of the ovary have recently reported a wide range of expression for CDX2 in this group of tumors (64-100\%). ${ }^{11,12,24,25}$ Such a heterogeneous CDX2 expression is intriguing since all four series used the same antibody clone in their analysis. In order to explain the lack of consistency in the literature on the expression of CDX2 in ovarian mucinous tumors, Gaggero et $a l^{26}$ evaluated the expression of CDX2 in 50 benign mucinous cystadenomas of the ovary (47 endocervical type and three intestinal types). The authors found all 47 endocervical-type mucinous cystadenomas stained negative for CDX2 and two of three intestinal-type cystadenomas stained positive. They concluded that the expression of CDX2 in mucinous tumors was likely dependent on the cell type (endocervical or intestinal). In the current study, two of the three mucinous tumors that expressed CDX2 were of pure intestinal type and the remainder was a mixed endocervical/intestinal subtype. We therefore agree with Gaggero et $a l^{26}$ that an attempt to correlate the CDX2 staining with the cell type may be helpful in understanding the wide range of reported expression of CDX2 in this group of tumors.

Recent morphologic observations have led to a reappraisal of the range of histologic findings that can be observed in metastatic tumors to the ovary, particularly from the gastrointestinal tract. ${ }^{2,3,19,27,28}$ It is well recognized that the morphologic mimicry of a metastatic tumor may be such that it may be extremely difficult to correctly ascribe a primary site in the absence of unequivocal clinical or histologic evidence of a known extraovarian primary. Two recent studies have further emphasized that the true 
incidence of primary mucinous carcinoma of the ovary may be significantly lower than that historically determined as a result of misclassification of metastatic tumors as ovarian primaries. ${ }^{27,29}$ In view of these findings, positive CDX2 expression in a mucinous tumor involving the ovary should be interpreted with caution; although it may be a primary ovarian adenocarcinoma, the possibility of a metastatic tumor needs to be carefully excluded. Conversely, negative expression of CDX2 in a mucinous tumor in the ovary would strongly support an ovarian primary since virtually all colonic adenocarcinomas express CDX2 in a diffuse pattern. In the current study, the five cases of primary ovarian carcinoma with diffuse CDX2 staining likely represent true enteric differentiation in these tumors at the genetic level and it would be of interest to study this selected group of ovarian tumors for genetic mutations involving the CDX2 gene.

$\alpha$-Methylacyl-CoA racemase, also known as P504S, is a mitochondrial and peroxisomal enzyme involved in the metabolism of fatty acids. ${ }^{17,30,31}$ Overexpression of P504S has been observed in several tumors, most notably prostate and colorectal carcinoma and in one study, $92 \%$ of colorectal carcinomas were found to overexpress this protein. ${ }^{16,32}$ The expression of this protein in cancer precursor lesions such as high-grade prostatic intraepithelial neoplasia and colorectal adenoma suggests that it has a role in early carcinogenesis. ${ }^{15}$ The expression of this protein in ovarian carcinoma has not been formally studied and limited information is available on its expression profile in epithelial tumors of the ovary of different histologic subtypes. In this study, $32 \%$ of metastatic colonic adenocarcinomas to the ovary showed diffuse expression of P504S as compared to none of the primary ovarian tumors. Jiang et $a l^{32}$ evaluated the expression of P504S in numerous neoplasms and found that $7 \%$ of ovarian adenocarcinomas expressed this protein, although the histologic subtype of ovarian adenocarcinoma was not provided and colonic adenocarcinomas were not evaluated. In all, $83 \%$ of the colonic adenocarcinomas evaluated in the study by Zhou et $a{ }^{16}$ demonstrated positive P504S protein expression. The higher percentage of cases of colonic adenocarcinoma with positive expression of this protein in the study by Zhou et $a l^{16}$ compared to the current study may be explained by the fact that we evaluated P504S expression in a selected group of metastatic tumors. There is some suggestion that the protein expression of P504S is related to the grade of the tumor with the highest expression in a tubular adenoma and decreased expression in high-grade tumors. ${ }^{15}$ Since metastatic tumors are more likely to be high grade, the decreased expression of P504S in the metastatic colorectal carcinomas in our study is not surprising.

A frequently observed genetic change in colorectal carcinoma is an inactivating mutation of the adeno- matous polyposis coli (APC) gene. ${ }^{33}$ This leads to abnormal accumulation of the protein $\beta$-catenin in the nucleus since the wild-type APC is involved in the degradation of this protein. ${ }^{34} \beta$-Catenin is a transactivating factor for the transcriptional factor Tcf-4 that regulates the expression of downstream target genes believed to be responsible for the oncogenic effect of a dysregulated $\beta$-catenin. ${ }^{35}$ Another mechanism for an abnormal accumulation of this protein is the presence of a direct mutation in the $\beta$-catenin gene with similar biologic effect. Since greater than $80 \%$ of colorectal carcinomas are thought to harbor a mutation in the APC gene, immunohistochemical detection of this protein in the tumor cell nucleus is a useful marker for colorectal carcinoma and is indicative of activation of the Wnt pathway through mutations in the APC or $\beta$-catenin pathway. Chou et $a l^{36}$ found nuclear expression of $\beta$-catenin in $83 \%$ of the colorectal carcinoma and $9 \%$ of ovarian mucinous tumors. Wang et $a l^{37}$ found $81 \%$ of colorectal carcinomas involving the urinary bladder to express $\beta$-catenin. Our results are comparable (59\%), although diffuse $(>2+)$ expression was observed in $36 \%$ of colonic and in only one of the ovarian tumors in our study. This case merits further discussion as it was an endometrioid adenocarcinoma with foci of borderline endometrioid tumor. In addition to strong and diffuse nuclear expression for $\beta$-catenin, a similar pattern of expression for CDX2 was also noted in this tumor. These patterns viewed in isolation strongly suggest a metastasis from a colonic tumor; however, after 3 years no colonic primary was clinically identified and the presence of borderline endometrioid neoplasia (rarely simulated by metastasis in contrast to the situation with mucinous tumors) indicated a likely primary nature. This case exemplifies that on rare occasions, CDX2 and $\beta$ catenin may be expressed by a primary ovarian adenocarcinoma in a pattern typical of a colorectal tumor. We have not performed genetic analysis on this case to determine if mutations in the aforementioned genes were present in this case or not, however, immunohistochemically the presence of a mutation is strongly suggested.

In summary, the immunohistochemical expression of specific markers of colorectal adenocarcinoma in mucinous and endometrioid adenocarcinoma of the ovary indicates that the phenotypic mimicry in these tumors extends to the genetic level in some cases. A panel comprising of CDX2, $\beta$-catenin and P504S is helpful in distinguishing a primary mucinous or endometrioid adenocarcinoma from a colorectal metastasis to the ovary in the majority of cases and is a useful adjunct to the already established role of differential staining with CK7/ CK20 in this differential diagnosis. Strong and diffuse nuclear expression of CDX2 along with diffuse nuclear expression of $\beta$-catenin favors a colorectal primary and expression of all three markers in a tumor is diagnostic of the same. 


\section{References}

1 Ulbright TM, Roth LM, Stehman FB. Secondary ovarian neoplasia. A clinicopathologic study of 35 cases. Cancer 1984;53:1164-1174.

2 Daya D, Nazerali L, Frank GL. Metastatic ovarian carcinoma of large intestinal origin simulating primary ovarian carcinoma. A clinicopathologic study of 25 cases. Am J Clin Pathol 1992;97:751-758.

3 Lee KR, Young RH. The distinction between primary and metastatic mucinous carcinomas of the ovary: gross and histologic findings in 50 cases. Am J Surg Pathol 2003;27:281-292.

4 Wauters CC, Smedts F, Gerrits LG, et al. Keratins 7 and 20 as diagnostic markers of carcinomas metastatic to the ovary. Hum Pathol 1995;26:852-855.

5 Albarracin CT, Jafri J, Montag AG, et al. Differential expression of MUC2 and MUC5AC mucin genes in primary ovarian and metastatic colonic carcinoma. Hum Pathol 2000;31:672-677.

6 Berezowski K, Stastny JF, Kornstein MJ. Cytokeratins 7 and 20 and carcinoembryonic antigen in ovarian and colonic carcinoma. Mod Pathol 1996;9:426-429.

7 Loy TS, Calaluce RD, Keeney GL. Cytokeratin immunostaining in differentiating primary ovarian carcinoma from metastatic colonic adenocarcinoma. Mod Pathol 1996;9:1040-1044.

8 Lagendijk JH, Mullink H, Van Diest PJ, et al. Tracing the origin of adenocarcinomas with unknown primary using immunohistochemistry: differential diagnosis between colonic and ovarian carcinomas as primary sites. Hum Pathol 1998;29:491-497.

9 Moll R, Lowe A, Laufer J, et al. Cytokeratin 20 in human carcinomas. A new histodiagnostic marker detected by monoclonal antibodies. Am J Pathol 1992;140:427-447.

10 Chu P, Wu E, Weiss LM. Cytokeratin 7 and cytokeratin 20 expression in epithelial neoplasms: a survey of 435 cases. Mod Pathol 2000;13:962-972.

11 Barbareschi M, Murer B, Colby TV, et al. CDX-2 homeobox gene expression is a reliable marker of colorectal adenocarcinoma metastases to the lungs. Am J Surg Pathol 2003;27:141-149.

12 Werling RW, Yaziji H, Bacchi CE, et al. CDX2, a highly sensitive and specific marker of adenocarcinomas of intestinal origin: an immunohistochemical survey of 476 primary and metastatic carcinomas. Am J Surg Pathol 2003;27:303-310.

13 Moskaluk CA, Zhang H, Powell SM, et al. Cdx2 protein expression in normal and malignant human tissues: an immunohistochemical survey using tissue microarrays. Mod Pathol 2003;16:913-919.

14 Hugh TJ, Dillon SA, O'Dowd G, et al. Beta-catenin expression in primary and metastatic colorectal carcinoma. Int J Cancer 1999;82:504-511.

15 Kuefer R, Varambally S, Zhou M, et al. alphaMethylacyl-CoA racemase: expression levels of this novel cancer biomarker depend on tumor differentiation. Am J Pathol 2002;161:841-848.

16 Zhou M, Chinnaiyan AM, Kleer CG, et al. Alpha-methylacyl-CoA racemase: a novel tumor marker over-expressed in several human cancers and their precursor lesions. Am J Surg Pathol 2002;26: 926-931.

17 Young RH, Scully RE. Metastatic tumors in the ovary: a problem-oriented approach and review of the recent literature. Semin Diagn Pathol 1991;8:250-276.
18 Arnell PM VQ, Eliva O. Beta-catenin expression in mucinous carcinomas of the ovary and metastatic colon carcinomas to the ovary: does it have a diagnostic role? Mod Pathol 2002;15:191A.

19 Ronnett BM, Kurman RJ, Shmookler BM, et al. The morphologic spectrum of ovarian metastases of appendiceal adenocarcinomas: a clinicopathologic and immunohistochemical analysis of tumors often misinterpreted as primary ovarian tumors or metastatic tumors from other gastrointestinal sites. Am J Surg Pathol 1997;21:1144-1155.

20 Lash RH, Hart WR. Intestinal adenocarcinomas metastatic to the ovaries. A clinicopathologic evaluation of 22 cases. Am J Surg Pathol 1987;11:114-121.

21 Bonner CA, Loftus SK, Wasmuth JJ. Isolation, characterization, and precise physical localization of human CDX1, a caudal-type homeobox gene. Genomics 1995;28:206-211.

22 Drummond F, Putt W, Fox M, et al. Cloning and chromosome assignment of the human CDX2 gene. Ann Hum Genet 1997;61(Part 5): 393-400.

23 Walters JR, Howard A, Rumble HE, et al. Differences in expression of homeobox transcription factors in proximal and distal human small intestine. Gastroenterology 1997;113:472-477.

24 Fraggetta F, Pelosi G, Cafici A, et al. CDX2 immunoreactivity in primary and metastatic ovarian mucinous tumours. Virchows Arch 2003;443:782-786.

25 Groisman GM, Meir A, Sabo E. The value of Cdx2 immunostaining in differentiating primary ovarian carcinomas from colonic carcinomas metastatic to the ovaries. Int J Gynecol Pathol 2004;23:52-57.

26 Gaggero G, Sola S, Mora M, et al. [Expression of the cdx2 gene in benign intestinal-type mucinous ovarian tumors]. Pathologica 2003;95:185-191.

27 Seidman JD, Kurman RJ, Ronnett BM. Primary and metastatic mucinous adenocarcinomas in the ovaries: incidence in routine practice with a new approach to improve intraoperative diagnosis. Am J Surg Pathol 2003;27:985-993.

28 Young RH, Hart WR. Metastases from carcinomas of the pancreas simulating primary mucinous tumors of the ovary. A report of seven cases. Am J Surg Pathol 1989;13:748-756.

29 Seidman JD, Horkayne-Szakaly I, Haiba M, et al. The histologic type and stage distribution of ovarian carcinomas of surface epithelial origin. Int J Gynecol Pathol 2004;23:41-44.

30 Schmitz W, Fingerhut R, Conzelmann E. Purification and properties of an alpha-methylacyl-CoA racemase from rat liver. Eur J Biochem 1994;222: 313-323.

31 Amery L, Fransen M, De Nys K, et al. Mitochondrial and peroxisomal targeting of 2-methylacyl-CoA racemase in humans. J Lipid Res 2000;41:17521759.

32 Jiang Z, Fanger GR, Woda BA, et al. Expression of alpha-methylacyl-CoA racemase (P504s) in various malignant neoplasms and normal tissues: a study of 761 cases. Hum Pathol 2003;34:792-796.

33 Miyoshi Y, Nagase H, Ando H, et al. Somatic mutations of the APC gene in colorectal tumors: mutation cluster region in the APC gene. Hum Mol Genet 1992;1: 229-233.

34 Behrens J, von Kries JP, Kuhl M, et al. Functional interaction of beta-catenin with the transcription factor LEF-1. Nature 1996;382:638-642. 
35 Morin PJ, Sparks AB, Korinek V, et al. Activation of beta-catenin-Tcf signaling in colon cancer by mutations in beta-catenin or APC. Science 1997;275: 1787-1790.

36 Chou YY, Jeng YM, Kao HL, et al. Differentiation of ovarian mucinous carcinoma and metastatic colorectal adenocarcinoma by immunostaining with beta-catenin. Histopathology 2003;43:151-156.

37 Wang HL, Lu DW, Yerian LM, et al. Immunohistochemical distinction between primary adenocarcinoma of the bladder and secondary colorectal adenocarcinoma. Am J Surg Pathol 2001;25:1380-1387. 